\title{
FLOOZY of petunia is a flavin mono-oxygenase-like protein required for the specification of leaf and flower architecture
}

\author{
Rafael Tobeña-Santamaria, ${ }^{1,3}$ Mattijs Bliek, ${ }^{1}$ Karin Ljung, ${ }^{2}$ Göran Sandberg, ${ }^{2}$ Joseph N.M. Mol, ${ }^{1}$ \\ Erik Souer, ${ }^{1}$ and Ronald Koes ${ }^{1,4}$ \\ ${ }^{1}$ Department of Developmental Genetics, Institute for Molecular Biological Sciences, Vrije Universiteit, Biocentrum \\ Amsterdam, 1081 HV Amsterdam, The Netherlands; ${ }^{2}$ Department of Forest Genetics and Plant Physiology, Swedish \\ University of Agricultural Sciences, S901 83 Umeå, Sweden
}

The mechanisms that determine the relative positions of floral organs, and thereby their numbers, is a poorly understood aspect of flower development. We isolated a petunia mutant, floozy (fzy), in which the formation of floral organ primordia in the outermost three floral whorls and one of the two bracts at the base of the flower is blocked at an early stage. In addition, fzy mutants fail to generate secondary veins in leaves and bracts and display a decreased apical dominance in the inflorescence. FZY encodes an enzyme with homology to flavin mono-oxygenases and appears to be the ortholog of YUCCA genes of Arabidopsis. FZY is expressed in young leafs and bracts and in developing flowers. In young floral meristems FZY is expressed in the center of the meristem dome and, later, expression becomes localized on the flanks of the initiating petal and stamen primordia and at several sites in maturing anthers and carpels. These findings indicate that FZY is involved in synthesizing a signaling compound that is required for floral organ initiation and specification of the vascularization pattern in leaves. Although $\mathrm{fzy}$ mutants contain normal auxin levels, ectopic expression of FZY results in excessive auxin accumulation, suggesting that the signaling compound is auxin.

[Key Words: Flavin mono-oxygenase; meristem initiation; flower development; vascularization; leaf development; transposon tagging]

Received October 30, 2001; revised version accepted January 18, 2002.

Flowers develop from groups of undifferentiated cells, floral meristems (FMs), that derive from the inflorescence meristem (IFM) located at the apex of the flowering shoot. The first dedicated step in flower development is the expression of meristem identity genes, such as LEAFY (LFY) and APETALA1 (AP1) in Arabidopsis (Mandell et al. 1992; Weigel et al. 1992) and homologs in other species (Coen et al. 1990; Huijser et al. 1992; Hofer et al. 1997; Kyozuka et al. 1998; Souer et al. 1998; MolineroRosales et al. 1999). In the absence of meristem identity gene activity, FMs remain fully or partially as IFMs, the apparent default pathway.

FMs differ from IFMs in the pattern of organ initiation and the identity of the organs that are formed; IFMs usually generate primordia for bracts and new FMs in a spiral pattern, whereas FMs generate floral organ primordia

\footnotetext{
${ }^{3}$ Present address: Department of Plant Physiology, University of Amsterdam, Kruislaan 318, 1098 SM Amsterdam, The Netherlands. ${ }^{4}$ Corresponding author.

E-MAIL koes@bio.vu.nl; FAX 31-2047155.

Article and publication are at http://www.genesdev.org/cgi/doi/10.1101/ $\operatorname{gad} 219502$.
}

in concentric whorls that develop into sepals, petals, stamens, and carpels. By mutation analysis, a number of genes that specify the identity of floral organs were identified (Coen and Meyerowitz 1991; Weigel and Meyerowitz 1994; Jack 2001), and at least some of these organ identity genes appear to be directly activated by meristem identity genes (Busch et al. 1999). However, the determination of the position and number of organ primordia within the floral meristem has remained a poorly understood aspect of the patterning of flowers (Running and Hake 2001).

The plant hormone auxin was recently implicated to play a key role in patterning of embryos, leaves, shoots, and roots and the initiation of a variety of meristems and primordia. Auxin metabolism is complex; the hormone can be synthesized by several distinct pathways, and free auxin can be inactivated by conjugation (Normanly and Bartel 1999|. Auxin synthesis takes place primarily in the shoot apex, and from there, it is transported downward by a polar transport system, which involves auxin influx and efflux carriers localized at the cell membrane (for review, see Palme and Galweiler 1999). 
Experiments in which the transport of auxin was blocked or reduced, either by treatment with chemical inhibitors or by mutations in genes encoding auxin transporters, inhibited the formation of leaf primordia and floral meristems at the shoot apex /Okada et al. 1991; Galweiler et al. 1998; Reinhardt et al. 2000; Vernoux et al. 2000), the initiation of lateral roots (Casimiro et al. 2001), the organization of the root meristem (Sabatini et al. 1999), embryo development (Okada et al. 1991; Liu et al. 1993; Steinmann et al. 1999), and the differentiation of vascular tissues (Berleth and Sachs 2001). However, the blocking of auxin transport may result in accumulation of excess auxin in some tissues (e.g., the unknown site of synthesis) and depletion elsewhere (Sabatini et al. 1999; Casimiro et al. 2001) and, moreover, chemical transport inhibitors not only affect the intracellular localization of auxin transporters, but also of other proteins (Geldner et al. 2001). Therefore, the role of auxin cannot be directly inferred from such auxin transport inhibition phenotypes. Blocked initiation of lateral roots, leafs, and flowers in transport inhibited (ex)plants can be overcome by (local) application of auxin (Reinhardt et al. 2000; Casimiro et al. 2001), indicating that, in these cases, the blocked organ initiation is due to auxin depletion.

Here, we report the molecular analysis of a petunia mutant, floozy $(f z y)$, in which the initiation of floral organ primordia in the outer three flower whorls is blocked at an early stage, whereas in leaves, secondary veins are not formed. FZY encodes a flavin mono-oxygenase-like protein that is the ortholog of YUCCA from Arabidopsis, and ectopic expression results in increased auxin levels and a phenotype similar to auxin-overproducing plants. Our data indicate that FZY functions in the synthesis of a hormone-like signaling compound, possibly auxin, that is required for the initiation of floral organ primordia and for the differentiation of secondary veins in leaves.

\section{Results}

\section{Isolation of the floozy mutant}

The petunia line W138 contains over 200 copies of the 284-bp transposon $d T p h 1$ (Gerats et al. 1990), and their frequent transposition causes a high incidence of mutations among W138 progeny (van Houwelingen et al. 1998). In a random transposon mutagenesis experiment, we screened large numbers of W138 progenies and identified a mutant, called floozy $\left(f_{z y}\right)$, in which the architecture of leaves and flowers was dramatically changed.

$f z y$ mutants display retarded growth and have shortened internodes compared with wild type (data not shown). Furthermore, the leaves are slightly wider than wild-type leaves, are often curled-up, and have an aberrant venation pattern. Wild-type leaves contain a central primary vein that branches into several secondary veins, which, in turn, branch into a dense network of tertiary and quaternary veins (Fig. 1A). $f z y$ leaves contain a central vein and a fine network of small veins that resemble the tertiary and quaternary veins in wild type, but secondary veins are missing, and the two lateral veins that run along the leaf margin are longer and more pronounced than in wild type (Fig. 1B). In mature fzy leaves, the region between the central and the lateral veins frequently turns yellow, presumably as a result of water and nutrient transport problems caused by the absence of secondary veins.

Wild-type petunia has a cymose inflorescence that is generated by repeated bifurcations of the inflorescence apex, resulting in the formation of multiple flowers on a zigzag-shaped inflorescence stem, (Fig. 1C,F; Souer et al. 1998). At the base of each flower, two bracts are found, each with a dormant (vegetative) axillary meristem in its axil. A normal flower contains five sepals, five petals, five stamens, and two carpels arranged in four concentric whorls (Fig. 1F,H). fzy mutants lack normal flowers and instead bear multiple structures that each consist of a pistil and a sepal-like organ on a petiole-like stem, whereas all petals, all stamens, and at least four of the five sepals are missing (Fig. 1G,I). At the base of this $f z y$ flower only a single bract, which is positioned opposite of the flower-like structure, and two axillary vegetative meristems are found (Fig. 1D,I). Because both axillary meristems are in their normal position, we believe that the sepal-like organ that is associated with the pistil in fzy flowers represents the first of the five sepals that are formed in wild type, rather than a displaced bract. The axillary meristems in $f z y$ inflorescences are much less dormant than those in wild-type inflorescences (Fig. $1 \mathrm{D}, \mathrm{G})$, and their vigorous growth is presumably the reason that activity of the apical IFM usually ceases after the production of 2-4 fzy flowers. The axillary meristems produce first 2-3 leaves and subsequently a few fzy flowers after which axillary meristems take over the growth, and the same sequence of events is initiated again. The continued reiteration of this program results in a very compact structure bearing relatively few flowers.

To test our interpretation of the $f z y$ inflorescence phenotype, we examined whether $f z y$ flowers are indeed equivalent to wild-type flowers by analysis of fzy double mutants with aberrant leaf and flower (alf) and extrapetals $(\exp )$. ALF is the petunia ortholog of $L F Y$ from Arabidopsis, and the alf inflorescence is a repeatedly bifurcated structure bearing only bracts, but no flowers, because FMs fail to adopt their identity and develop as IFMs instead (Fig. 1J; Souer et al. 1998). fzy, alf double mutants bear leaves and bracts with the aberrant venation pattern seen in $f z y$ single mutants (data not shown), and their inflorescence consists of a bifurcated structure that lacks the typical $f z y$ flowers consisting of a sepallike organ and a pistil (Fig. $1 \mathrm{~K}$ ). Interestingly, the $f z y$, alf double mutant inflorescence carries two, and sometimes three, bracts at each bifurcation point, just like alf single mutants, suggesting that the formation of the bract that is missing in a $f z y$ single mutant is restored again in the $f z y$, alf double mutants. The EXP gene is required for bifurcation of the inflorescence apex into an FM and an IFM, and, consequently, the exp inflorescence consists of a single flower (Souer et al. 1998; Fig. 1L). exp, fzy double 

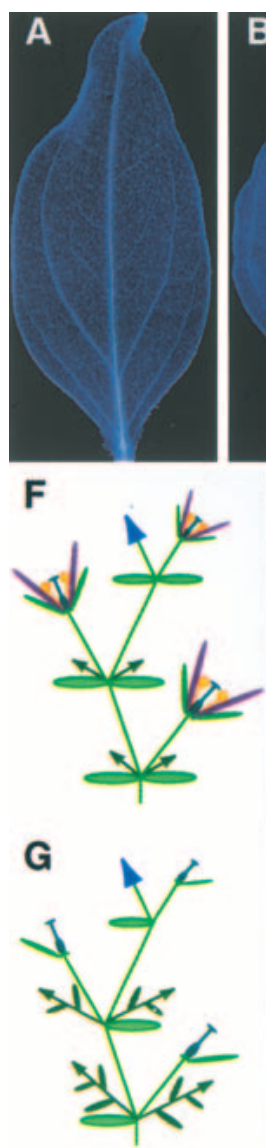
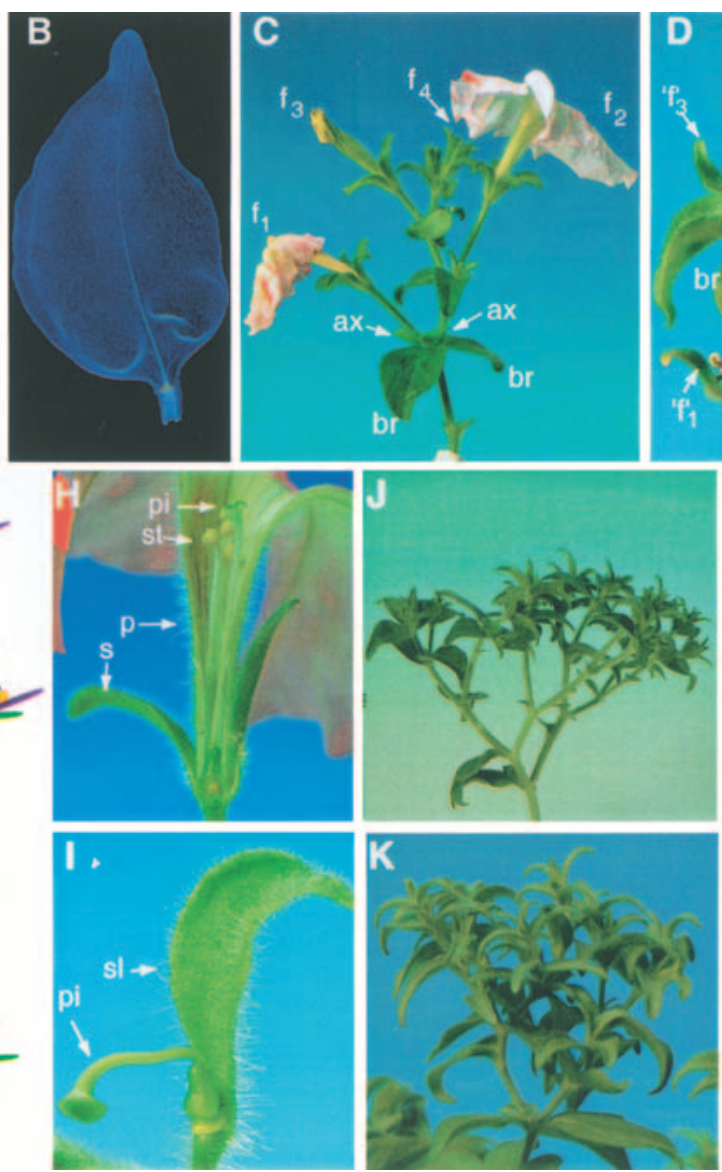
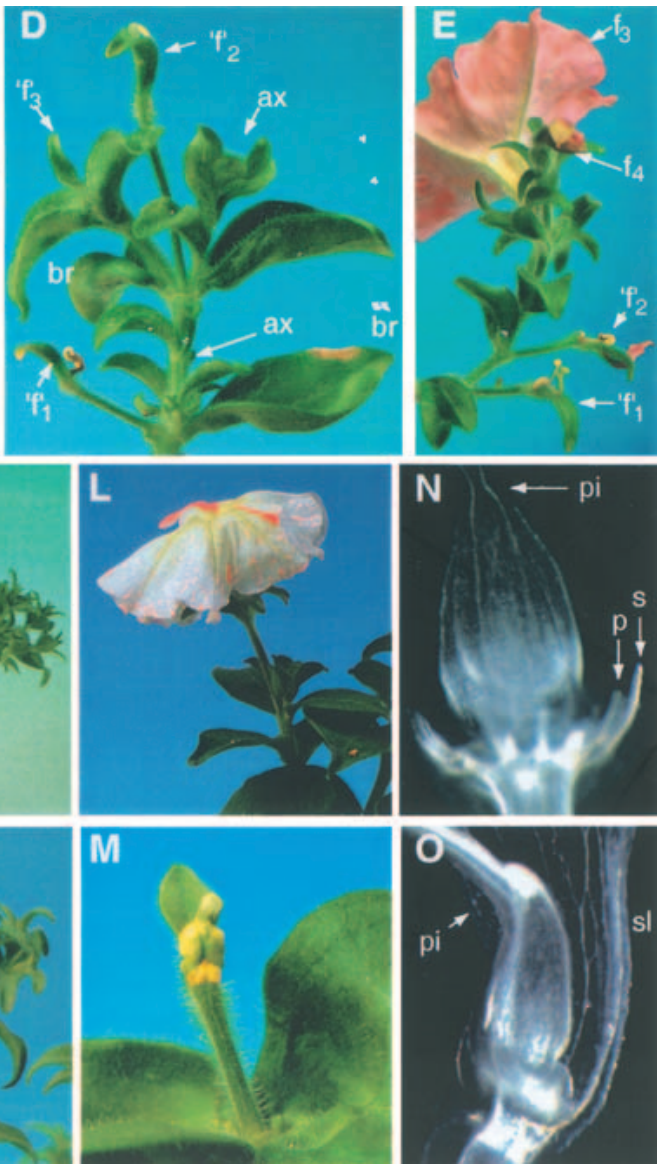

Figure 1. Analysis of the $f z y$ phenotype. $(A)$ Cleared leaf of a wild-type plant. $(B)$ Cleared leaf of a $f z y$ plant. $(C)$ Inflorescence of wild-type plant. Note that, at the base of the flowers (f1-f4 from old to young), two bracts (br) are found with a dormant axillary meristem $(\mathrm{ax})$ in their axils. $(D)$ Inflorescence of a fzy mutant plant. Note that, at the base of the flower-like structures (' $\mathrm{f}$ '), only one bract $(\mathrm{br})$ is found as well as two axillary meristems $(\mathrm{ax})$ with reduced dormancy. $(E)$ Somatic reversion of $f_{z y}$ in an inflorescence branch. Note that the oldest flowers (' $f$ ' 1 and ' $f$ ' 2 ) contain one stamen and a piece of petal tissue respectively, whereas the youngest flowers (f3 and f4) are nearly perfect and that the axillary meristems that accompany each of these flowers are dormant. $(F)$ Diagram of the wild-type inflorescence and $(G)$ a $f z y$ inflorescence. Bracts are indicated as horizontal green ovals and the axillary meristems as dark green triangles, with leaves (dark green ovals) when they are not dormant. (H) Flower of a wild-type plant consisting of sepals (s), petals (p), stamens (st) and a pistil (pi). (I) Flower-like structure on a fzy mutant consisting of a sepal-like organ (sl) and a pistil (pi). (J) Inflorescence of an alf mutant and $(K)$ of an $f z y$, alf double mutant. Note that bracts have been removed from the lower part of the inflorescence in $J$ to reveal the branching pattern. $(L)$ Inflorescence of an exp single mutant. $(M)$ Inflorescence of an exp, $f z y$ double mutant. $(N)$ Detail of cleared flowers of wild type. (O) Detail of cleared flowers of a $f z y$ mutant. For clarity, petals $(\mathrm{p})$ and sepals $(\mathrm{s})$ were almost completely removed in $N$.

mutants bear only one fzy flower per branch (Fig. 1M), whereas their leaves have again the typical $f z y$ venation pattern. Therefore, we conclude that the fzy-specific structure consisting of the pistil and a sepal-like organ is the equivalent of a flower.

To examine the aberrations in fzy flowers in further detail, we analyzed the anatomy of wild-type and fzy flowers by serial sectioning (data not shown) and by whole-mount analysis of cleared flowers. These experiments showed that, in wild-type plants, the ring-shaped structure of vascular bundles in the pedicel diverges in the flower bottom (receptacle) into numerous branches that invade the floral organs (Fig. 1N). The vascular bundles running in $f z y$ flowers are normal in the pedicel, but, in the receptacle, they collapse and no (attempted) branching is seen (Fig. 1O). Thus, mature fzy flowers completely lack (remnants of) floral organs in the outer three whorls.

The pistil of $f z y$ flowers was infertile (pollination with wild-type pollen never resulted in seed set) and displayed structural aberrations. The vasculature of the carpels was abnormal (data not shown), and usually one of the two carpels, and sometimes both, was strongly reduced in growth resulting in a strongly curved style. In addition, the number of ovules was reduced compared with wild type (data not shown).

FZY is required for the initiation of organ primordia, but not for patterning of the floral meristem

To analyze early stages of $f z y$ flower development, we examined dissected inflorescence apices and flowers by 
scanning electron microscopy. The dome of a wild-type apical IFM first generates primordia for two bracts and then splits into two distinct domes (Fig. 2A; Souer et al. 1998). The most apical dome, the FM, subsequently enlarges and sequentially generates five sepals in a slightly asynchronous fashion (Fig. 2A). The first recognizable sepal primordium arises opposite of the IFM (the rightmost sepal in Fig. 2A), after which the primordia for the other sepals arise as two pairs. Once the last two sepal primordia are well established, the primordia for petals, stamens, and carpels arise sequentially in the more inside whorls of the flower (Fig. 2B). Note that, at these very early stages of flower development, the initiation of the axillary meristems at the base of the flower is not visible yet.

In $f z y$ mutants, the bifurcation of the IFM still occurs (Fig. 2C), but the FM does not enlarge as wide as in wild type and is almost completely used for the formation of two carpel primordia. The formation of the carpel primordia was preceded by the initiation of the sepal-like organ, which, on the basis of its position relative to the IFM, appears to represent the first arising sepal in wild
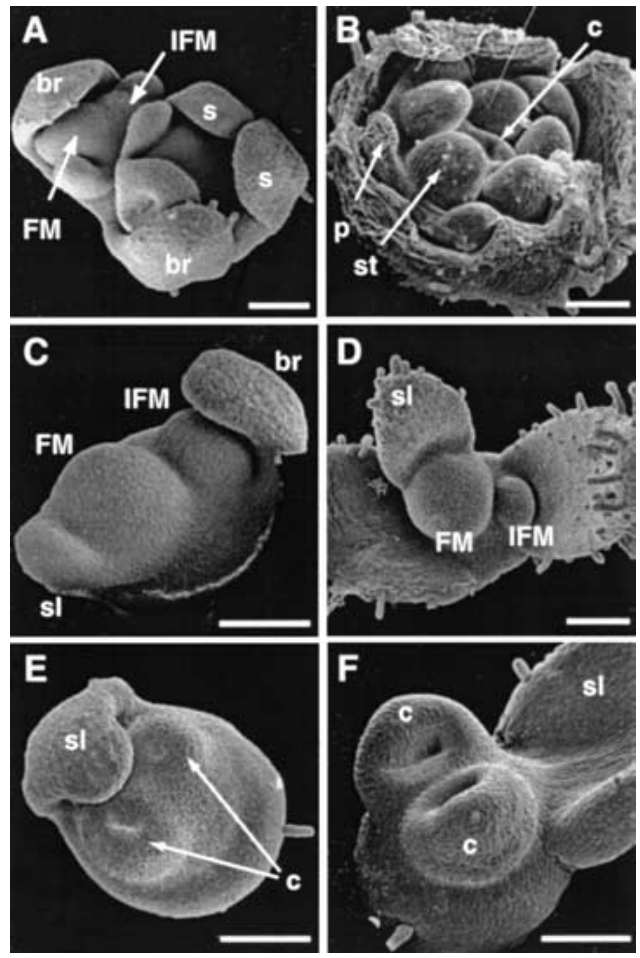

Figure 2. Early stages of flower development in wild type and fzy. (A) Scanning electron micrograph of a wild-type inflorescence apex with a young (stage 3) FM that arose by bifurcation of the IFM, and a young flower (stage 5) that has already formed five sepals (s), but not yet visible primordia in the inner whorls. (B) Wild-type flower (stage 7) showing the whorled arrangement of primordia for petals $(\mathrm{p})$, stamens (st), and carpels $(\mathrm{c}) .(C-F)$ Scanning electron micrographs of $f z y$ inflorescence and flowers showing the initiating bract (br), the bifurcation of the IFM and the FM, and the primordium for the sepal-like-organ (sl) in $C$ and $D$, and the formation of carpel primordia (c) in $E$ and $F$. Bars, $100 \mu \mathrm{m}$.

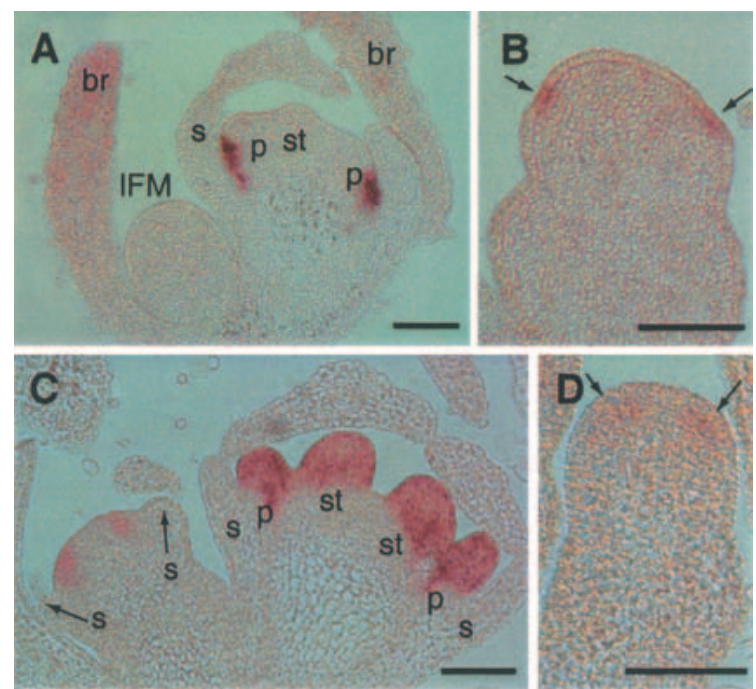

Figure 3. Expression of $D O T$ and $F B P 1$ in wild-type and $f z y$ floral meristems. (A) DOT expression in a FZY flower. (B) DOT expression (arrows) in $f z y$ flower. $(C) F B P 1$ expression in FZY flowers. (D) FBP1 expression (arrows) in $f z y$ flower. (IFM) Inflorescence meristem; (br) bract; (s) sepal; (p) petal; (st) stamen. Bars, $100 \mu \mathrm{m}$.

type. However, no initiation of primordia for the other four sepals, which arise slightly later in wild type, or any of the anthers and petals was observed (Fig. 2D-F).

To assess whether the formation of patterns within the $f z y$ FMs was altered, we analyzed the expression of the meristem identity gene DOUBLE TOP (DOT) and the organ identity gene FLORAL BINDING PROTEIN1 (FBP1). DOT is the petunia ortholog of UNUSUAL FLORAL ORGANS (UFO) of Arabidopsis and is, together with $A L F$, required to specify FM identity (E. Souer, M. Bliek, C. van Schie, R. de Bruin, J. Mol, and R. Koes, in prep.). In young FMs of wild type, DOT is expressed in a ring of cells at the border of the whorls 1 and 2 (Fig. 3A; E. Souer, M. Bliek, C. van Schie, R. de Bruin, J. Mol, and R. Koes, in prep.). fzy FMs contain a ring of DOT-expressing cells in roughly the same position, but the DOT mRNA levels are much lower than in corresponding wild-type cells (Fig. 3B). FBP1 is a B-type organ identity gene that is required to specify the identity of petal and stamens (Angenent et al. 1995). In wild-type FMs FBP1 is expressed in a wide ring of cells at the site where whorl two and three primordia will arise (Fig. 3C). In fzy FMs FBP1 is expressed in a similar domain, albeit very weakly (Fig. 3D).

Together, these results indicate that the fzy FM is still correctly patterned, that is, the formation and interpretation of positional cues is not abolished, but that the initiation of organ primordia is impaired at an early stage.

\section{FZY function is non cell-autonomous}

The (transposon-tagged) $f z y-V 2022$ allele occasionally produced somatically reverted branches bearing nearly 
wild-type flowers. As an example, Figure 1E shows an inflorescence branch in which a somatic reversion occurred. In the two oldest flowers (' $f$ ' 1 and ' $f$ ' 2 ) only a single anther (in ' $\mathrm{f}$ ' 1 ) and a streak of petal tissue (in ' $\mathrm{f}$ '2) is formed, whereas the next two flowers (f3 and f4) are almost completely restored to wild type, indicating that one of the three tunica layers of the apical IFM has been almost entirely taken over by descendants of a FZY revertant cell between the formation of the FMs from which flowers ' $\mathrm{f}$ ' 2 and $\mathrm{f} 3$ developed. The axillary meristems at the base of the mutant flowers ' $\mathrm{f}$ ' 1 and ' $\mathrm{f}$ ' 2 , however, are dormant as in wild type. Because these axillary meristem developed much later than ' $\mathrm{f}$ ' 1 and ' $\mathrm{f}$ '2 and because ' $\mathrm{f}$ ' 1 and ' $\mathrm{f}$ ' 2 are almost completely mutant, we assume that the restored dormancy of their axillary meristems depends on the restored FZY activity in the more acropetal tissues (near f3 and f4).

Cross-pollination of FZY flowers from the same revertant branch resulted at low frequency in seed set (14 out of $>100$ pollinations), indicating that fertility was partially restored. In four of these cases, the original reversion was genetically transmissible as the progeny segregated 3:1 for FZY and $f z y$ plants, indicating that the reversion had occurred in a cell in the L2 tunica layer of the apical meristem (from which the gametes originate). In 10 other cases, the reversion was not transmissible, and the resulting progenies consisted entirely of $f z y$ plants, indicating that the reversion had occurred in either the L1 or the L3 tunica layer. Because the development of L1-, L2-, and L3-derived floral tissues was restored to a similar extent in $\mathrm{L} 2$ and L1/L3 revertant branches, FZY appears to function in a non cell-autonomous manner.

\section{Molecular isolation of fzy}

To isolate FZY, we analyzed $d T p h 1$ transposons, the major cause of spontaneous mutations in petunia (van Houwelingen et al. 1998), by transposon-display (van den Broek et al. 1998) and identified a 98-bp fragment that contained a $d T p h 1$ insertion in eight $f z y-V 2022$ plants, but not in plants homozygous for the parental wild-type or a revertant allele (Fig. 4A). Sequencing of corresponding cDNA and genomic fragments showed that it originated from a gene, consisting of four exons, that in $f z y$ $V 2022$ plants was disrupted by a $d T p h 1$ insertion in exon 3. PCR experiments showed that the FZY progeny from (L2) revertant branches lacked this $d T p h 1$ insertion in one or both alleles of the isolated gene (Fig. 4B). However, because these revertant alleles lacked a transposon footprint, we could not fully exclude the remote possibility that they resulted from pollen contamination, rather than transposon excision.

To obtain full proof for the identity of the isolated gene, we screened 4000 petunia plants by a PCR-based assay (Koes et al. 1995) and identified six heterozygous plants that harbored new alleles, in which a $d T p h 1$ transposon had inserted into the protein-coding sequence (four alleles) or in an intron (two alleles; Fig. 4C). Progeny obtained by self-fertilization of heterozygotes for the
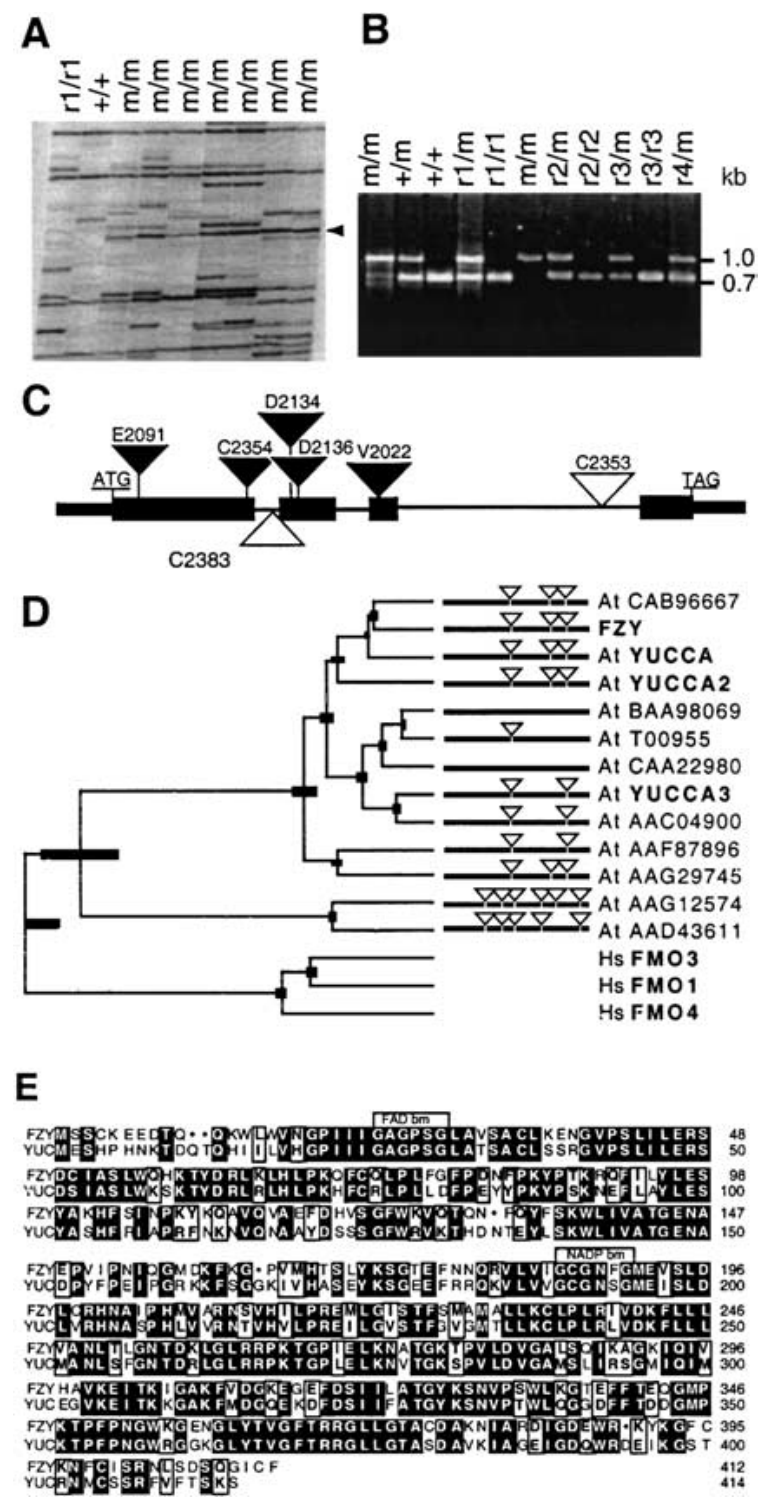

Figure 4. Molecular analysis of FZY. (A) Transposon display of plants homozygous for the mutable $f z y-V 2022(\mathrm{~m} / \mathrm{m})$, the progenitor $(+/+)$ or a revertant allele $(r 1 / r 1)$. (Arrow) 98-bp $f z y$ fragment. (B) PCR amplification of a FZY gene fragment from plants harboring $f z y-V 2022(\mathrm{~m})$, the wild-type progenitor $(+)$, and four independent revertant alleles $(r 1-r 4)$. (C) Structure of the FZY gene and mutant alleles. (Closed boxes and thin lines) Exons and introns, respectively; (open and closed inverted triangles) $d T p h 1$ insertions in FZY and $f z y$ alleles, respectively. $(D)$ Tree constructed by unweighted pair method using arithmetic averages (UPGMA) showing the similarity between FZY and FMO-like proteins from Arabidopsis (At) and humans (Hs). (Thick bars) Uncertainty of the branch point. The diagrams indicate the positions of introns (triangles) and exons (horizontal bars). Sequences are named after the protein (bold) or GenBank accession number. (E) Alignment of FZY and YUCCA showing the position of putative binding motifs for FAD and NADP.

intron insertions C2383 and C2353 consisted of wildtype $\left(F Z Y^{+}\right)$plants only. This result came as no surprise, because insertions of the small (284 bp) dTph1 element in introns only rarely block gene expression (C. Spelt, E. 
Souer, F. Quattrocchio, and R. Koes, unpubl.). However, self-fertilization of heterozygotes harboring the exon insertions C2354, D2134, D2136, and E2091 gave progenies that segregated 3:1 for wild type and $f z y$ mutants. Subsequent complementation tests showed that these mutants were all allelic to the original $f z y$-V2022 mutant (data not shown). Therefore, we concluded that the isolated gene is FZY.

Sequence analysis of the FZY cDNA clone showed that it encodes a 412 amino acid protein (GenBank accession no. AY039108) with extensive similarity to flavin mono-oxygenases (FMOs) and FMO-like proteins from mammals, fungi, and plants and contains conserved binding motifs for flavin-adenine dinucleotide (FAD) and reduced nicotinamide adenine dinucleotide phosphate (NADPH; Fig. 4D,E). The highest similarity was found with a family of nine Arabidopsis genes, including YUCCA1, YUCCA2, YUCCA3, and six genes encoding FMO-like proteins of unknown function (GenBank accession nos. CAB96667, BAA98069, T00955, CAA22980, AAC04900, and AAF87896; Fig. 4D), whereas a second Arabidopsis family of 11 putative FMO-like proteins (in Fig. 4D represented by the GenBank accession nos. AAG12574 and AAD43611) has much lower similarity.

To determine which of these Arabidopsis FMO-like proteins represented the true ortholog of FZY, we compared the intron/exon structure of the genes. Figure $4 \mathrm{~A}$ shows that the genes CAB96667, YUCCA, YUCCA2, and AAG29745 contain three introns in exactly the same position as the three introns in FZY. In the other five members of this subfamily, one or more of these introns were missing; whereas the genes AAG12574 and AAD43611, belonging to the second subfamily of genes encoding FMO-like proteins, have introns in completely different positions. Taken together, these findings indicate that FZY is the petunia ortholog of YUCCA1, YUCCA2, and CAB96667 of Arabidopsis.

\section{Role of FZY in auxin synthesis}

Recent gain-of-function experiments showed that ectopic expression of YUCCA1 or YUCCA3 in Arabidopsis and tobacco resulted in overproduction of auxin and that Escherichia coli-produced YUCCA could catalyze the conversion of tryptamine into N-hydroxyl tryptamine, a step in a putative auxin pathway (Zhao et al. 2001).

To test whether FZY is involved in the synthesis of auxin, we measured the amounts of free indole acetic acid (IAA), the major auxin, in wild type and $f z y$ mutants. However, we did not find a clear reduction of the amount of free auxin in vegetative apices or young leaves of $f z y$ plants (Fig. 5A), even though these tissues express fzy mRNA (see below). Analysis of five T2 progeny plants originating from a transgenic petunia line that ectopically (over)expresses FZY from a transgene driven by the strong and constitutive $35 \mathrm{~S}$ promoter of Cauliflower Mosaic Virus, showed that they contained increased auxin levels in the apical region (i.e., the shoot apex plus the first leaves up to $\sim 0.5 \mathrm{~cm}$ in length) and the
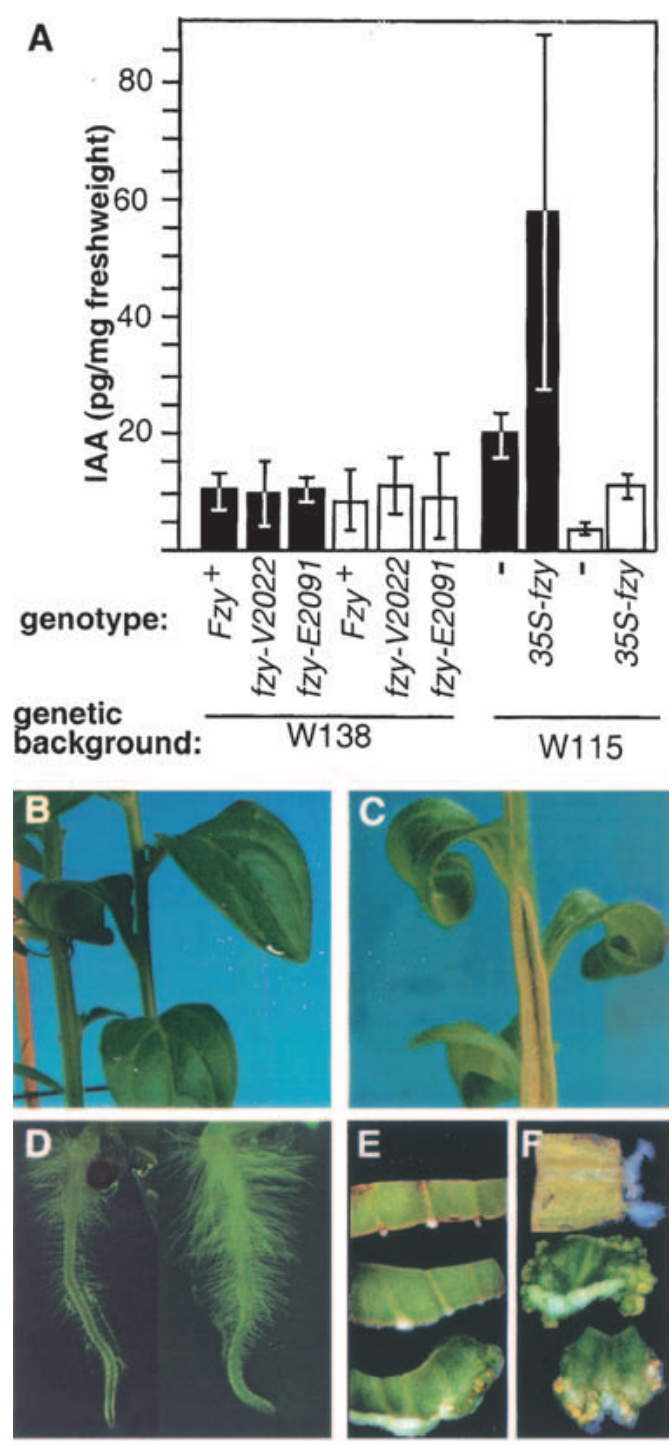

Figure 5. Auxin levels in $f z y$ mutants. (A) Concentrations (mean \pm standard deviation) of free indole acetic acid (IAA) in apices (containing the SAM and several leaves up to a size of $\sim 5$ $\mathrm{mm})$ or the tip of a young leaf $(\sim 2 \mathrm{~cm})$ of 4 -week-old vegetative plants are indicated by filled and open bars respectively. (B) Stem and leaves of a wild-type W115 plant and of $(C)$ a W115 plant containing a 35S-FZY transgene. Note the bursted, woody stem in the latter. $(D)$ Roots of a W115 seedling (left) and a W115::35S::FZY seedling. $(E, F)$ Explants of mock transformed W115 plants $(E)$ or W115 plants harboring a 35S::FZY transgene $(F)$, grown for $6 \mathrm{~d}$ on MS medium (top), MS medium containing $5 \mu \mathrm{M}$ zeatin (middle), or $5 \mu \mathrm{M}$ zeatin and $0.5 \mu \mathrm{M}$ naphthalene acetic acid (NAA) (bottom).

tip of young $(\sim 2 \mathrm{~cm})$ leaves (Fig. 5A). The relatively large variation in auxin levels in these plants correlated with the strength of the phenotype (see below) and presumably resulted from variations in transgene expression level.

The 35S::FZY plants displayed several aberrations compared with wild type, such as long, narrow, epinastic leaves, a hard and woody stem (Fig. 5, cf. B and C), and 
more and longer root hairs (Fig. 5D), similar to auxinoverproducing Arabidopsis (Boerjan et al. 1994; Barlier et al. 2000; Bak et al. 2001; Zhao et al. 2001), tobacco (Zhao et al. 2001), and petunia plants (Klee et al. 1987). Furthermore, explants from $35 S$ ::FZY plants displayed reduced auxin dependency in tissue culture (Fig. 5E,F). Leaf explants of nontransgenic W115 plants require naphthalene acetic acid (NAA; an auxin) and zeatin (a cytokinin) to stimulate callus formation; in the absence of either one, only a small amount of callus is formed in the first days after transfer to MS-medium. Explants of 35S::FZY plants readily formed callus in the presence of zeatin alone, indicating that they had become auxin independent. In the absence of both NAA and zeatin, roots were formed, albeit at a low frequency (Fig. 5F), which is indicative of a high internal auxin to cytokinin ratio.

Together, these data indicate that FZY functions in an auxin pathway, that wild-type plants synthesize only a minor fraction of the total auxin pool. However, we cannot fully exclude the possibility that the extra auxin synthesized in FZY (and YUCCA) overexpressors is due to relaxed substrate specificity and that in vivo FZY is involved in the synthesis of a distinct signaling molecule (see Discussion).

\section{Expression pattern of $\mathrm{fzy}$}

To identify which tissues synthesize the putative auxin signal, we analyzed the expression pattern of FZY in wild-type plants by in situ hybridization (Fig. 6). During the vegetative phase, FZY mRNA is detectable in young leaves in a thin layer of cells in the center of the blade (Fig. 6A,B), whereas the shoot apical meristem (SAM) is devoid of detectable $F Z Y$ expression. In inflorescences, $F Z Y$ is expressed in bracts, in a similar pattern as in leaves (Fig. 6C). In FMs, FZY mRNA was detected from the earliest stages on, initially in a small region in the center of the FM anlagen (Fig. 6D). During subsequent growth of the FM, the FZY expression domain widens somewhat (Fig. 6E) and opens in the center (Fig. 6F). At stage 5, FZY expression becomes restricted to rings of tissue surrounding the now well visible stamen and petal primordia, whereas in the further advanced sepals, FZY is expressed in a central layer of cells, similar to leaves and bracts (Fig. 6G,H). In stage 8 flowers, FZY expression is limited to a layer of cells in the center of the petal blade, a superficial region at the distal end of the placenta, and a narrow band of cells at the circumference of anthers at the position where the stomium will appear later (Fig. 6I-L).

Together, these data show that the FZY expression pattern correlates well with the defects seen in fzy mutants, indicating that even though FZY acts non cellautonomously, its action is short range.

\section{Discussion}

We report the identification of the FZY gene of petunia, which is required for initiation of organs in the outer-
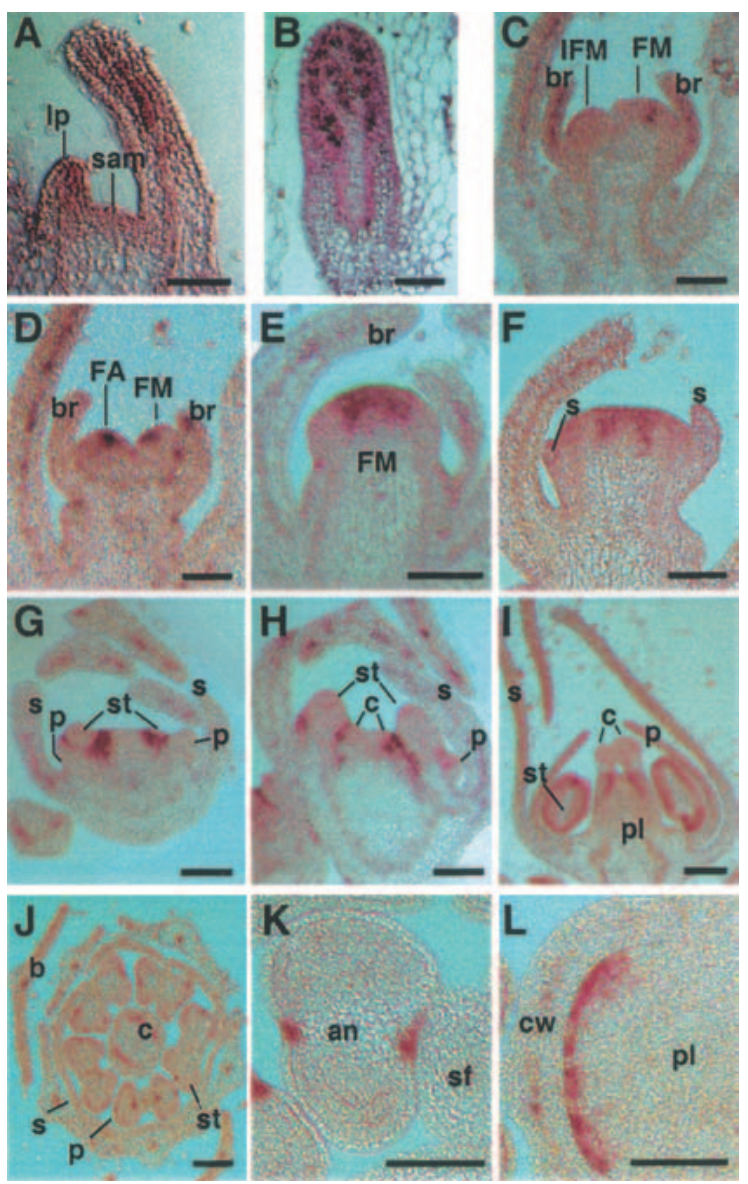

Figure 6. In situ localization of FZY mRNA in wild-type plants. (A) Section through a vegetative apex. $(B)$ Mediolateral section through the blade of a leaf. $(C)$ Section through an inflorescence apex, showing FZY expression in stage 4 FM and bracts and absence of expression in the IFM. (D) Different section from the same apex as in $C$, showing FZY expression in the flower anlagen ( stage 1). (E-I) Sections through flowers of stages $3(E), 4(F), 5(G), 6(H)$, and $8(I)$. $(J-K)$ Cross-sections through a stage 8 flower. (lp) Leaf primordium; (br) bract; (FA) flower anlage; (s) sepal; (p) petal; (st) stamen; (c) carpel; (pl) placenta; (an) anther; (sf) stamen filament; $(\mathrm{cw})$ carpel wall. Bars, $250 \mu \mathrm{m}$ in $I$ and $J$ and $100 \mu \mathrm{m}$ in all other panels.

most three whorls of the flower and for the specification of the vascularization pattern in leaves and bracts. FZY encodes an enzyme with strong homology to flavin mono-oxygenases and appears to be the petunia ortholog of YUCCA genes of Arabidopsis. Combined with the observation that FZY function is not fully cell-autonomous, it is suggestive that FZY is involved in the synthesis of a signaling compound that is required for floral organ initiation and leaf patterning.

\section{Nature of the end product of the FZY pathway}

The high similarity of FZY to YUCCA1 and YUCCA2 of Arabidopsis, both in sequence and in gene structure (Fig. 4 ), as well as their highly similar ectopic (over)expression 
phenotypes, indicate that these proteins are orthologs and catalyze similar reactions. Ectopic expression of either YUCCA genes in Arabidopsis or FZY in petunia results in increased auxin levels, narrow and epinastic leaves, more and longer root hairs and auxin-independent growth in tissue culture (Zhao et al. 2001; Fig. 5), which are well known characteristics of auxin overproduction (Klee et al. 1987; Boerjan et al. 1994; Barlier et al. 2000; Bak et al. 2001). Furthermore, it was shown (Gil et al. 2001) that ectopic YUCCA expression can restore the defects caused by reduced auxin transport in the aerial part of tir3 mutants (Ruegger et al. 1997), and that the ectopic YUCCA (over)expression phenotype can be reversed by expression of a bacterial enzyme (IAA-L) that inactivates free IAA by conjugating it to lysine (Zhao et al. 2001).

Although these data indicate that the ectopic expression phenotype of YUCCA and FZY is due to excess auxin, they do not exclude that the effect on auxin synthesis is indirect. In this respect, it is noteworthy that mutations in the Arabidopsis gene CYP79F1 (also known as BUSHY [BUS] and SUPERSHOOT [SPS]), encoding a cytochrome P450 involved in the synthesis of short chain glucosinolates (Hansen et al. 2001), not only abolished short chain glucosinolates, but also increased the levels of cytokinins and IAA by a mechanism that is not understood (Reintanz et al. 2001; Tantikanjana et al. 2001). Biochemical experiments showed that a YUCCAmaltose binding protein (MBP) fusion protein produced in E. coli could catalyze the conversion of tryptamine into $\mathrm{N}$-hydroxyl tryptamine, a step in a putative auxin pathway, suggesting that YUCCA is directly involved in auxin synthesis (Zhao et al. 2001). However, because $\mathrm{K}_{\mathrm{m}}$ values were not determined, and the amounts of YUCCA-MBP protein that were used in these experiments was very high, it is difficult to judge whether this activity of YUCCA is of significance in vivo. The finding that free IAA levels are increased in $35 S:: F Z Y$ overexpressors, but not significantly decreased in $f z y$ mutants may, therefore, be explained in two ways. First, the $f z y$ phenotype may be due to very local auxin deficiency, and the bulk of the auxin in apical tissues is synthesized by a FZY-independent pathway. Second, at physiological FZY concentrations, the major product of the FZY pathway may be another compound and the role of FZY in auxin synthesis is negligible, whereas the auxin-synthesizing potential of FZY only becomes evident at high protein concentrations.

To discriminate between these possibilities we tested whether application of exogenous auxin could rescue the $f z y$ phenotype. Repeated application of auxin solutions over a 3-week period onto flowering $f z y$ apices did not result in a restoration of floral organ formation above the background (apices treated with water) or the formation of secondary veins in bracts. However, these negative results are difficult to interpret, because, in the absence of a positive control, it is difficult to assess whether the applied auxin reaches the young meristems and flower primordia, which are buried between more mature bracts and floral structures.

\section{Role of FZY in inflorescence development}

Although the direct evidence for a role of FZY and YUCCA in auxin synthesis is strong, but not water tight, we note that the $f z y$ loss-of-function phenotype is compatible with a role of FZY in a localized (minor) auxin synthesis pathway. First, the reduced apical dominance in the inflorescence (Fig. 1D,G) may be due to a reduced auxin production in the young $f z y$ flowers in more acropetal positions (Fig. 1E). Second, the defect in $f z y$ floral meristems, arrest of organ primordium initiation at an early stage, resembles the defects seen in Arabidopsis and tomato inflorescences treated with transport inhibitors or mutants in PIN1, PINOID (PID), or MONOPTEROS $(M P)$, in which an auxin efflux carrier (Okada et al. 1991; Galweiler et al. 1998), a kinase involved in auxin signaling (Christensen et al. 2000) or transport (Benjamins et al. 2001), and a transcription factor thought to act in auxin signaling (Hardtke and Berleth 1998) are disrupted, respectively. Such inflorescences consist of a naked pin (Przemeck et al. 1996; Hardtke and Berleth 1998), because FM initiation is blocked at a very early stage (Christensen et al. 2000; Vernoux et al. 2000), apparently because of local auxin depletion (Reinhardt et al. 2000). Mutations in PIN1 and PID affect FM initiation at the apex, but not the patterning (i.e., the setup or interpretation of positional cues) of the meristem (Christensen et al. 2000; Vernoux et al. 2000). Similarly, the organization of the young FM does not seem to be disturbed in $f z y$ mutants, because $f z y$ FMs still express DOT and FBP1 mRNA in a pattern similar to wild-type FMs (Figs. 2 and 3), although the mRNA levels of expression are much lower. Given that DOT and FBP1 are both involved in the specification of floral organ identity, it is indicative that the initiation of organ identity specification precedes and occurs independently from the early FZY-dependent step in organ initiation. However, the subsequent maintenance of organ identity (i.e., further up regulation of organ identity genes) seems inhibited by the $f z y$ mutation.

In $f z y$ mutants, the formation of the bract neighboring the FM is blocked, whereas the formation of the FM itself and the bract neighboring the IFM is still normally initiated (Fig. 1D,G). This finding, together with the observation that in an $f z y$, alf double mutants both bracts are formed, suggests that the function of FZY is partially redundant and that the activity of FZY in the young flower (anlagen) is mirrored by a FZY-like activity in or near the IFM. The latter activity may be responsible for the initiation of the FM, the first sepal, and the bract neighboring the IFM, whereas the initiation of the bract neighboring the FM apparently depends on FZY activity in the flower (anlagen). If so, the formation of the latter bract should be restored when the identity of the young FM meristem is converted into an IFM, by mutation of $A L F$, as now both meristems will express the FZY-like activity. These events are indeed observed (Fig. 1K).

At early stages of FM initiation, before organ primordia become anatomically visible, $f z y$ is expressed in the center of the FM (Fig. 6C-E) in a pattern that is very 
similar to that of PIN1, PID, and MP (Hardtke and Berleth 1998; Christensen et al. 2000). Thus, the auxin that is transported and signaled by these proteins, might well be derived from FZY expression in the same and nearby cells. At later stages, $F Z Y$ expression becomes restricted to the boundaries of petals and stamens (Fig. 6G,H) whereas PIN1, PID, and MP continue to be expressed in the primordia themselves. At this stage, the FZY expression pattern shows a striking resemblance to that of $N O$ APICAL MERISTEM (NAM), a gene that is required to restrict the proliferation of primordia in whorls 2 and 3 of the flower, (Souer et al. 1996), suggesting that in wildtype FMs, FZY (and derived auxin) does not block NAM expression. This suggestion seems to contrast the results obtained with pin inflorescences, which suggested that one role of auxin during the initiation of FMs is to inhibit expression of CUC2, the Arabidopsis ortholog of NAM (Aida et al. 1997), in the center of the emerging FM (Vernoux et al. 2000). One reason for such apparently conflicting results is that it is difficult, if not impossible, to infer the actual distribution pattern of a hormone (the active compound) from the expression patterns of genes involved in its synthesis or transport.

\section{Role of FZY in vascularization of leaves}

Although the mechanisms that determine the vascularization pattern in leaves are still largely unknown (for review, see Dengler and Kang 2001), a range of experiments implicated a key role for auxin (for review, see Berleth et al. 2000). For instance, local application of auxin can induce vascularization in a narrow region extending basipetally from the site of application, suggesting that polar auxin transport is involved in determining the directionality of the response (Sachs 1991). Consistent with this, treatment of Arabidopsis leaves with auxin transport inhibitors drastically alters venation pattern, confining the veins to the leaf margin (Mattsson et al. 1999; Sieburth 1999). Mutations in PIN1, MP, and LOP1, which affect auxin transport and/or signaling, also reduce vascularization, but, for reasons that are not understood, the venation patterns of these mutants are very different (Carland and McHale 1996; Przemeck et al. 1996; Mattsson et al. 1999). Auxin inactivation by expression of the bacterial IAA-L gene reduces xylem formation, but, perhaps surprisingly, has no clear effect on venation pattern (Romano et al. 1991).

In $f z y$ mutants, the differentiation of secondary veins in leaves and bracts is blocked (Fig. 1A), which in itself is consistent with a role of FZY in auxin synthesis. In young leaves and bracts, FZY is expressed in a central layer of cells more or less throughout the leaf blade (Fig. 5 ), and the expression pattern appears somewhat speckled with some single cells stained more intensely than others (Fig. 5A). In older leaves, bracts, and sepals, this speckled expression pattern becomes more and more pronounced (Fig. 5C-I). It seems likely that these regions of FZY-expressing cells, coincide with the sites where secondary veins are differentiating, but definite proof will require demonstration that FZY expression colocal- izes with that of an early marker for vascular differentiation, such as, for example, the ATHB gene of Arabidopsis (Baima et al. 1995; Dengler and Kang 2001).

At present, it is unclear why fzy affects specifically the formation of secondary veins, without a clear effect on primary or tertiary and quaternary veins. Given that the functions of the orthologous YUCCA genes in Arabidopsis are highly redundant (Zhao et al. 2001) and that primary, secondary, and tertiary veins arise sequentially (for review, see Dengler and Kang 2001), it is possible that differentiation of primary and tertiary veins depends on other (partially) redundant genes with a different spatiotemporal expression pattern. Further information on this issue will require the analysis of mutations in all FZY or YUCCA paralogs.

\section{Materials and methods}

\section{Petunia lines}

The $f z y$-V2022 allele was identified in a family of W138 plants, and revertants were obtained by cross-pollination of flowers on the same revertant branch. Because of the pollination procedures used, and the reproduction characteristics of petunia (for details, see van Houwelingen et al. 1999) contamination with pollen from FZY plants can be virtually completely excluded.

Additional $d T p h 1$ insertion alleles were identified by PCR analysis of 48 DNA samples from leaves (pooled in a threedimensional matrix) of $\sim 4000$ Petunia W138 plants, using a primer complementary to $d T p h 1$ and various primers complementary to the coding sequence of FZY (Koes et al. 1995). Progenies of the five plants with $d T p h 1$ insertions in FZY exons segregated $f z y$ mutants, whereas progenies of the $\sim 2100$ other plants that were used for a next round of transposon mutagenesis consisted entirely of FZY plants. Subsequent crosses of heterozygotes harboring different $f z y$ alleles, resulted in progenies that segregated 3:1 for wild type and $f z y$ mutants, confirming that all mutants represented alleles of the same gene.

The mutant alleles alf-W2167 and exp-W2115 were also isolated in the W138 background and have been described in detail before (Souer et al. 1998).

For ectopic expression of FZY, the cDNA was ligated in between the 35S promoter of Cauliflower Mosaic Virus and the polyadenylation signal of the NOPALINE SYNTHASE gene (NOS) and introduced into petunia line W115 (also known as Mitchell) by Agrobacterium-mediated transformation, as described before (Spelt et al. 2000).

\section{Microscopy}

Tissues were cleared for detailed anatomical analyses under a dissection microscope by soaking in acetic acid:ethanol (6:1) until all chlorophyll was removed and then in chloral hydrate: water:glycerol $(8: 2: 1)$ until they became fully transparent. In situ hybridization analysis and scanning electron microscopy was carried out as described previously (Souer et al. 1996). To detect transcripts of DOT and FZY, RNA probes complementary to the entire coding sequence were used. For detection of FBP1 mRNA, we used a probe hybridizing to an mRNA region well downstream of the conserved MADS box (region +367 to +740 relative to the ATG start codon) Floral stages were numbered according to Maes et al. (2001). 


\section{DNA methodology}

To display $d T p h 1$ flanking sequences, genomic DNA was cut with MseI, ligated to an MseI adapter oligonucleotide as described (van den Broek et al. 1998) and PCR-amplified, first using primers complementary to the MseI adapter and $d T p h 1$, and then, in a second round, by an MseI adapter primer extended with a C, A, T, or G nucleotide and a nested ${ }^{33} \mathrm{P}$-labeled primer complementary to $d T p h 1 .{ }^{33} \mathrm{P}$-labeled fragments were separated on $6 \%$ denaturing polyacrylamide (sequencing) gels and visualized by PhosphorImaging.

A 98-bp fragment of $f z y$ was isolated from the display gel, (re)amplified by PCR, cloned into a plasmid, and used to screen a cDNA library made from inflorescence apices of FZY W138 plants. The genomic FZY fragment was obtained by PCR with primers complementary to the $5^{\prime}$ - and $3^{\prime}$-untranslated regions in the cDNA clone.

\section{Auxin measurements}

Auxin levels were determined by gas chromatography and mass spectrometry (GC-MS) as described (Edlund et al. 1995).

\section{Acknowledgments}

We thank Saskia Kars for assistance with scanning electron microscopy, Pieter Hoogeveen, Martina Meesters, and Daisy Kloos for their care of the plants and Fred Schuurhof for photographic work. This work was supported by an EU Marie Curie fellowship to R.T. and grant support by the Netherlands Technology Foundation (S.T.W.), with financial aid from the Netherlands Organisation for the Advancement of Research (N.W.O.).

The publication costs of this article were defrayed in part by payment of page charges. This article must therefore be hereby marked "advertisement" in accordance with 18 USC section 1734 solely to indicate this fact.

\section{References}

Aida, M., Ishida, T., Fukaki, H., Fujisawa, H., and Tasaka, M. 1997. Genes involved in organ separation in Arabidopsis: An analysis of the cup-shaped cotyledon mutant. Plant Cell 9: $841-857$.

Angenent, G.C., Bussher, M., Franken, J., Dons, H.J.M., and van Tunen, A.J. 1995. Functional interaction between the homeotic genes $f b p 1$ and $p M A D S 1$ during petunia floral organ organogenesis. Plant Cell 7: 507-516.

Baima, S., Nobili, F., Sessa, G., Lucchetti, S., Ruberti, I., and Morelli, G. 1995. The expression of the Athb-8 homeobox gene is restricted to provascular cells in Arabidopsis thaliana. Development 121: 4171-4182.

Bak, S., Tax, F.E., Feldmann, K.A., Galbraith, D.W., and Feyereisen, R. 2001. CYP83B1, a cytochrome P450 at the metabolic branch point in auxin and indole glucosinolate biosynthesis in Arabidopsis. Plant Cell 13: 101-111.

Barlier, I., Kowalczyk, M., Marchant, A., Ljung, K., Bhalerao, R., Bennett, M., Sandberg, G., and Bellini, C. 2000. The SUR2 gene of Arabidopsis thaliana encodes the cytochrome P450 CYP83B1, a modulator of auxin homeostasis. Proc. Nat1. Acad. Sci. 97: 14819-14824.

Benjamins, R., Quint, A., Weijers, D., Hooykaas, P., and Offringa, R. 2001. The PINOID protein kinase regulates organ development in Arabidopsis by enhancing polar transport. Development 128: 4057-4067.

Berleth, T. and Sachs, T. 2001. Plant morphogenesis: Long-dis- tance coordination and local patterning. Curr. Opin. Plant Biol. 4: 57-62.

Berleth, T., Mattsson, J., and Hardtke, C.S. 2000. Vascular continuity and auxin signals. Trends Plant Sci. 5: 387-393.

Boerjan, W., Bauw, G., Van Montagu, M., and Inze, D. 1994. Distinct phenotypes generated by overexpression and suppression of S-adenosyl-L-methionine synthetase reveal developmental patterns of gene silencing in tobacco. Plant Cell 6: 1401-1414.

Busch, M.A., Bomblies, K., and Weigel, D. 1999. Activation of a floral homeotic gene in Arabidopsis. Science 285: 585-587.

Carland, F.M. and McHale, N.A. 1996. LOP1: A gene involved in auxin transport and vascular patterning in Arabidopsis. Development 122: 1811-1819.

Casimiro, I., Marchant, A., Bhalerao, R., Beeckman, T., Dhooge, S., Swarup, R., Graham, N., Inzé, D., Sandberg, G., Casero, P.J., et al. 2001. Auxin promotes Arabidopsis lateral root initiation. Plant Cell 13: 843-852.

Christensen, S.K., Dagenais, N., Chory, J., and Weigel, D. 2000. Regulation of auxin response by the protein kinase PINOID. Cell 100: 469-478.

Coen, E.S. and Meyerowitz, E.M. 1991. The war of the whorlsGenetic interactions controlling flower development. $\mathrm{Na}$ ture 353: 31-37.

Coen, E.S., Roemro, J.M., Doyle, S., Elliott, R., Murphy, G., and Carpenter, R. 1990. Floricaula: A homeotic gene required for flower development in Antirrhinum majus. Cell 63: 13111322.

Dengler, N. and Kang, J. 2001. Vascular patterning and leaf shape. Curr. Opin. Plant Biol. 4: 50-56.

Edlund, A., Eklöf, S. Sundberg, B., Moritz, T., and Sandberg, G. 1995. A miscroscale technique for gas chromatography-mass spectrometry measurements of picogram amounts of indole3-acetic acid in plant tissues. Plant Physiol. 108: 1043-1047.

Galweiler, L., Guan, C., Müller, A, , Wisman, E., Mendgen, K., Yephremov, A., and Palme, K. 1998. Regulation of polar auxin transport by AtPIN1 in Arabidopsis vascular tissue. Science 282: 2226-2230.

Geldner, N., Friml, J., Stierhof, Y.D., Jurgens, G., and Palme, K. 2001. Auxin transport inhibitors block PIN1 cycling and vesicle trafficking. Nature 413: 425-428.

Gerats, A.G.M., Huits, H., Vrijlandt, E., Maraña, C., Souer, E., and Beld, M. 1990. Molecular characterization of a nonautonomous transposable element (dTph1) of petunia. Plant Cell 2: 1121-1128.

Gil, P., Dewey, E., Friml, J., Zhao, Y., Snowden, K.C., Putterill, J., Palme, K., Estelle, M., and Chory, J. 2001. BIG: A calossinlike protein required for polar auxin transport in Arabidopsis. Genes \& Dev. 15: 1985-1997.

Hansen, C.H., Wittstock, U., Olsen, C.E., Hick, A.J., Pickett, J.A., and Halkier, B.A. 2001. Cytochrome P450 CYP79F1 from Arabidopsis catalyzes the conversion of dihomomethionine and trihomomethionine to the corresponding aldoximes in the biosynthesis of aliphatic glucosinolates. $J$. Biol. Chem. 276: 11078-11085.

Hardtke, C.S. and Berleth, T. 1998. The Arabidopsis gene MONOPTEROS encodes a transcription factor mediating embryo axis formation and vascular development. EMBO $\mathrm{J}$. 17: 1405-1411.

Hofer, J., Turner, L., Hellens, R., Ambrose, M., Matthews, P., Michael, A., and Ellis, N. 1997. UNIFOLIATA regulates leaf and flower morphogenesis in pea. Curr. Biol. 7: 581-587.

Huijser, P., Klein, J., Lönnig, W.-E., Meijer, H., Saedler, H., and Sommer, H. 1992. Bractomania, an inflorescence anomaly, is caused by the loss of function of the MADS-box gene squamosa in Anthirrhinum. EMBO J. 11: 1239-1249. 
Jack, T. 2001. Relearning our ABCs: New twists on an old model. Trends Plant Sci. 6: 310-316.

Klee, H., Horsch, R.B., Hinchee, M.A., Hein, M.B., and Hoffman, N.L. 1987. The effects of overproduction of two Agrobacterium tumefaciens T-DNA auxin biosynthetic gene products in transgenic petunia plants. Genes \& Dev. 1: 8696.

Koes, R., Souer, E., van Houwelingen, A., Mur, L., Spelt, C., Quattrocchio, F., Wing, J.F., Oppedijk, B., Ahmed, S., Maes, T., et al. 1995. Targeted gene inactivation in petunia by PCRbased selection of transposon insertion mutants. Proc. Natl. Acad. Sci. 81: 8149-8153.

Kyozuka, J., Konishi, S., Nemoto, K., Izawa, T., and Shimamoto, K. 1998. Down-regulation of RFL, the FLO/LFY homolog of rice, accompanied with panicle branch initiation. Proc. Nat1. Acad. Sci. 95: 1979-1982.

Liu, C.-M., Xu, Z.-H., and Chua, N.-H. 1993. Auxin polar transport is essential for the establishment of bilateral symmetry during early plant embryogenesis. Plant Cell 5: 621-630.

Maes, T., Van de Steene, N., Zethof, N., Karimi, M., D'Hauw, M., Mares, G., Van Montagu, M., and Gerats, T. 2001. Petunia Ap2-like genes and their role in flower and seed development. Plant Cell 13: 229-244.

Mandell, M.A., Gustafson-Brown, C., Savidge, B., and Yanofsky, M. 1992. Molecular characterization of the Arabidopsis homeotic gene APETALA1. Nature 360: 273-277.

Mattsson, J., Sung, Z.R., and Berleth, T. 1999. Responses of plant vascular systems to auxin transport inhibition. Development 126: 2979-2991.

Molinero-Rosales, N., Jamilena, M., Zurita, S., Gomez, P., Capel, J., and Lozano, R. 1999. FALSIFLORA, the tomato orthologue of FLORICAULA and LEAFY, controls flowering time and floral meristem identity. Plant J. 20: 685-693.

Normanly, J. and Bartel, B. 1999. Redundancy as a way of lifeIAA metabolism. Curr. Opin. Plant Biol. 2: 207-213.

Okada, K., Ueda, J., Komaki, M., Bell, C., and Shimura, Y. 1991. Requirement of the auxin polar transport system in early stages of Arabidopsis floral bud formation. Plant Cell 3: 677-684.

Palme, K. and Galweiler, L. 1999. PIN-pointing the molecular basis of auxin transport. Curr. Opin. Plant Biol. 2: 375-381.

Przemeck, G.K., Mattsson, J., Hardtke, C.S., Sung, Z.R., and Berleth, T. 1996. Studies on the role of the Arabidopsis gene MONOPTEROS in vascular development and plant cell axialization. Planta 200: 229-237.

Reinhardt, D., Mandel, T., and Kuhlemeier, C. 2000. Auxin regulates the initiation and radial position of plant lateral organs. Plant Cell 12: 507-518.

Reintanz, B., Lehnen, M., Reichelt, M., Gershenzon, J., Kowalczyk, M., Sandberg, G., Godde, M., Uhl, R., and Palme, K. 2001. bus, a bushy Arabidopsis CYP79F1 knockout mutant with abolished synthesis of short-chain aliphatic glucosinolates. Plant Cell 13: 351-367.

Romano, C.P., Hein, M.B., and Klee, H.J. 1991. Inactivation of auxin in tobacco transformed with the indoleacetic acid-lysine synthetase gene of Pseudomonas savastanoi. Genes \& Dev. 5: 438-446.

Ruegger, M., Dewey, E., Hobbie, L., Brown, D., Bernasconi, P., Turner, J., Muday, G., and Estelle, M. 1997. Reduced naphthylphthalamic acid binding in the tir3 mutant of Arabidopsis is associated with a reduction in polar auxin transport and diverse morphological defects. Plant Cell 9: 745-757.

Running, M.P. and Hake, S. 2001. The role of floral meristems in patterning. Curr. Opin. Plant Biol. 4: 69-74.

Sabatini, S., Beis, D., Wolkenfelt, H., Murfett, J., Guilfoyle, T., Malamy, J., Benfey, P., Leyser, O., Bechtold, N., Weisbeek,
P., et al. 1999. An auxin-dependent distal organizer of pattern and polarity in the Arabidopsis root. Cell 99: 463-472.

Sachs, T. 1991. Cell polarity and tissue patterning in plants. Development Suppl. 91: 83-93.

Sieburth, L.E. 1999. Auxin is required for leaf vein pattern in Arabidopsis. Plant Physiol. 121: 1179-1190.

Souer, E., van Houwelingen, A., Kloos, D., Mol, J.N.M., and Koes, R. 1996. The no apical meristem gene of petunia is required for pattern formation in embryos and flowers and is expressed at meristem and primordia boundaries. Cell 85: $159-170$

Souer, E., van der Krol, A.R., Kloos, D., Spelt, C., Bliek, M., Mol, J., and Koes, R. 1998. Genetic control of branching pattern and floral identity during Petunia inflorescence development. Development 125: 733-742.

Spelt, C., Quattrocchio, F., Mol, J., and Koes, R. 2000. anthocyanin1 of petunia encodes a basic-helix loop helix protein that directly activates structural anthocyanin genes. Plant Cell 12: 1619-1631.

Steinmann, T., Geldner, N., Grebe, M., Mangold, S., Jackson, C.L., Paris, S., Galweiler, L., Palme, K., and Jurgens, G. 1999. Coordinated polar localization of auxin efflux carrier PIN1 by GNOM ARF GEF. Science 286: 316-318.

Tantikanjana, T., Yong, J.W., Letham, D.S., Griffith, M., Hussain, M., Ljung, K., Sandberg, G., and Sundaresan, V. 2001. Control of axillary bud initiation and shoot architecture in Arabidopsis through the SUPERSHOOT gene. Genes \& Dev. 15: 1577-1588.

van den Broek, D., Maes, T., Sauer, M., Zethof, J., De Keukelaire, P., D'Hauw, M., Van Montagu, M., and Gerats, T. 1998. Transposon display identifies individual transposable elements in high copy number lines. Plant J. 13: 121-129.

van Houwelingen, A., Souer, E., Mol, J.N.M., and Koes, R.E. 1999. Epigenetic interactions between three dTph1 transposons in two homologous chromosomes activates a new excision-repair mechanism in petunia. Plant Cell 11: 13191336.

van Houwelingen, A., Souer, E., Spelt, C., Kloos, D., Mol, J., and Koes, R. 1998. Analysis of flower pigmentation mutants generated by random transposon mutagenesis in Petunia hybrida. Plant J. 13: 39-50.

Vernoux, T., Kroneberger, J., Grandjean, O., Laufs, P., and Traas, J. 2000. PIN-FORMED 1 regulates cell fate at the periphery of the shoot apical meristem. Development 127: 5157-5165.

Weigel, D. and Meyerowitz, E.M. 1994. The ABCs of floral homeotic genes. Cell 78: 203-209.

Weigel, D., Alvarez, J., Smyth, D., Yanofsky, M.F., and Meyerowitz, E.M. 1992. LEAFY controls floral meristem identity in Arabidopsis. Cell 69: 843-859.

Zhao, Y., Christensen, S.K., Fankhauser, C., Cashman, J.R., Cohen, J.D., Weigel, D., and Chory, J. 2001. A role for flavin monooygenase-like enzymes in auxin biosynthesis. Science 291: 306-309. 


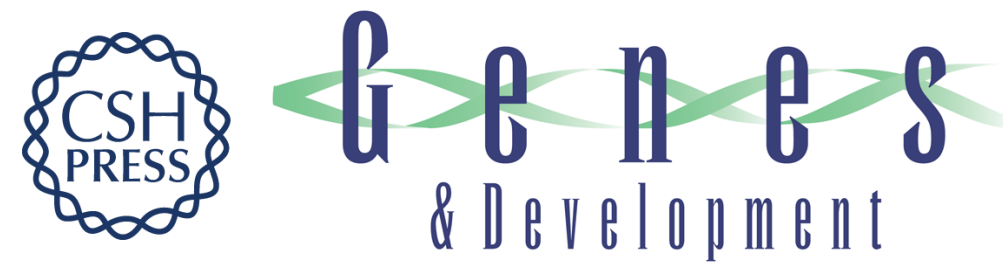

\section{FLOOZY of petunia is a flavin mono-oxygenase-like protein required for the specification of leaf and flower architecture}

Rafael Tobeña-Santamaria, Mattijs Bliek, Karin Ljung, et al.

Genes Dev. 2002, 16:

Access the most recent version at doi:10.1101/gad.219502

References This article cites 57 articles, 34 of which can be accessed free at: http://genesdev.cshlp.org/content/16/6/753.full.html\#ref-list-1

License

Email Alerting

Receive free email alerts when new articles cite this article - sign up in the box at the top Service right corner of the article or click here.

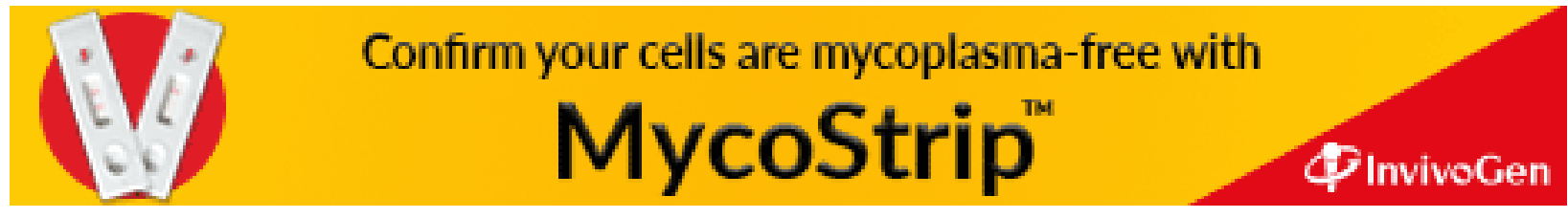

